\title{
Research Article \\ Fixed Point Theorems for ws-Compact Mappings in Banach Spaces
}

\author{
Ravi P. Agarwal, ${ }^{1,2}$ Donal O'Regan, ${ }^{3}$ and Mohamed-Aziz Taoudi ${ }^{4}$ \\ ${ }^{1}$ Department of Mathematical Sciences, Florida Institute of Technology, 150 West University Boulevard, \\ Melbourne, FL 32901, USA \\ ${ }^{2}$ Mathematics and Statistics Department, King Fahd University of Petroleum and Minerals, \\ Dhahran 31261, Saudi Arabia \\ ${ }^{3}$ Department of Mathematics, National University of Ireland, Galway, Ireland \\ ${ }^{4}$ Université Cadi Ayyad, Laboratoire de Mathématiques et de Dynamique de Populations, \\ Marrakech, Morocco
}

Correspondence should be addressed to Ravi P. Agarwal, agarwal@fit.edu

Received 17 August 2010; Revised 21 October 2010; Accepted 4 November 2010

Academic Editor: Jerzy Jezierski

Copyright (C) 2010 Ravi P. Agarwal et al. This is an open access article distributed under the Creative Commons Attribution License, which permits unrestricted use, distribution, and reproduction in any medium, provided the original work is properly cited.

We present new fixed point theorems for ws-compact operators. Our fixed point results are obtained under Sadovskii, Leray-Schauder, Rothe, Altman, Petryshyn, and Furi-Pera type conditions. An example is given to show the usefulness and the applicability of our results.

\section{Introduction}

Let $X$ be a Banach space, and let $M$ be a subset of $X$. Following [1], a map $A: M \rightarrow X$ is said to be ws-compact if it is continuous and for any weakly convergent sequence $\left(x_{n}\right)_{n \in \mathbb{N}}$ in $M$ the sequence $\left(A x_{n}\right)_{n \in \mathbb{N}}$ has a strongly convergent subsequence in $X$. This concept arises naturally in the study of both integral and partial differential equations (see [1-5]). In this paper, we continue the study of ws-compact mappings, investigate the boundary conditions, and establish new fixed point theorems. Specifically, we prove several fixed point theorems for ws-compact mappings under Sadovskii, Leray-Schauder, Rothe, Altman, Petryshyn and Furi-Pera type conditions. Finally, we note that ws-compact mappings are not necessarily sequentially weakly continuous (see Example 2.14). This explains the usefulness of our fixed point results in many practical situations. For the remainder of this section, we gather some notations and preliminary facts. Let $X$ be a Banach space, let $B(X)$ denote the collection of 
all nonempty bounded subsets of $X$ and $\mathcal{W}(X)$ the subset of $\mathcal{B}(X)$ consisting of all weakly compact subsets of $X$. Also, let $B_{r}$ denote the closed ball centered at 0 with radius $r$.

In our considerations, the following definition will play an important role.

Definition 1.1 (see [6]). A function $\psi: B(X) \rightarrow \mathbb{R}_{+}$is said to be a measure of weak noncompactness if it satisfies the following conditions.

(1) The family $\operatorname{ker}(\psi)=\{M \in B(X): \psi(M)=0\}$ is nonempty and $\operatorname{ker}(\psi)$ is contained in the set of relatively weakly compact sets of $X$.

(2) $M_{1} \subseteq M_{2} \Rightarrow \psi\left(M_{1}\right) \leq \psi\left(M_{2}\right)$.

(3) $\psi(\overline{\mathrm{CO}}(M))=\psi(M)$, where $\overline{\mathrm{CO}}(M)$ is the closed convex hull of $M$.

(4) $\psi\left(\lambda M_{1}+(1-\lambda) M_{2}\right) \leq \lambda \psi\left(M_{1}\right)+(1-\lambda) \psi\left(M_{2}\right)$ for $\lambda \in[0,1]$.

(5) If $\left(M_{n}\right)_{n \geq 1}$ is a sequence of nonempty weakly closed subsets of $X$ with $M_{1}$ bounded and $M_{1} \supseteq M_{2} \supseteq \cdots \supseteq M_{n} \supseteq \cdots$ such that $\lim _{n \rightarrow \infty} \psi\left(M_{n}\right)=0$, then $M_{\infty}:=\bigcap_{n=1}^{\infty} M_{n}$ is nonempty.

The family ker $\psi$ described in (1) is said to be the kernel of the measure of weak noncompactness $\psi$. Note that the intersection set $M_{\infty}$ from (5) belongs to ker $\psi$ since $\psi\left(M_{\infty}\right) \leq \psi\left(M_{n}\right)$ for every $n$ and $\lim _{n \rightarrow \infty} \psi\left(M_{n}\right)=0$. Also, it can be easily verified that the measure $\psi$ satisfies

$$
\psi\left(\overline{M^{w}}\right)=\psi(M)
$$

where $\overline{M^{w}}$ is the weak closure of $M$.

A measure of weak noncompactness $\psi$ is said to be regular if

$$
\psi(M)=0 \text { if and only if } M \text { is relatively weakly compact, }
$$

subadditive if

$$
\psi\left(M_{1}+M_{2}\right) \leq \psi\left(M_{1}\right)+\psi\left(M_{2}\right),
$$

homogeneous if

$$
\psi(\lambda M)=|\lambda| \psi(M), \quad \lambda \in \mathbb{R},
$$

set additive (or have the maximum property) if

$$
\psi\left(M_{1} \cup M_{2}\right)=\max \left(\psi\left(M_{1}\right), \psi\left(M_{2}\right)\right)
$$


The first important example of a measure of weak noncompactness has been defined by De Blasi [7] as follows:

$$
w(M)=\inf \left\{r>0: \text { there exists } W \in \mathcal{W}(X) \text { with } M \subseteq W+B_{r}\right\}
$$

for each $M \in B(X)$.

Notice that $w(\cdot)$ is regular, homogeneous, subadditive, and set additive (see [7]).

In what follows, let $X$ be a Banach space, $C$ a nonempty closed convex subset of $X$, $F: C \rightarrow C$ a mapping and $x_{0} \in C$. For any $M \subseteq C$, we set

$$
\begin{gathered}
F^{\left(1, x_{0}\right)}(M)=F(M), \\
F^{\left(n, x_{0}\right)}(M)=F\left(\overline{\mathrm{CO}}\left(F^{\left(n-1, x_{0}\right)}(M) \cup\left\{x_{0}\right\}\right)\right),
\end{gathered}
$$

for $n=2,3, \ldots$

Definition 1.2. Let $X$ be a Banach space, $C$ a nonempty closed convex subset of $X$, and $\psi$ a measure of weak noncompactness on $X$. Let $F: C \rightarrow C$ be a bounded mapping (that is it takes bounded sets into bounded ones) and $x_{0} \in C$. We say that $F$ is a $\psi$-convex-power condensing operator about $x_{0}$ and $n_{0}$ if for any bounded set $M \subseteq C$ with $\psi(M)>0$, we have

$$
\psi\left(F^{\left(n_{0}, x_{0}\right)}(M)\right)<\psi(M)
$$

Obviously, $F: C \rightarrow C$ is $\psi$-condensing if and only if it is $\psi$-convex-power condensing operator about $x_{0}$ and 1 .

Remark 1.3. The concept of convex-power condensing maps was introduced in [8] using the Kuratowski measure of noncompactness.

\section{Fixed Point Theorems}

Theorem 2.1. Let $X$ be a Banach space, and let $\psi$ be a regular and set additive measure of weak noncompactness on $X$. Let $C$ be a nonempty closed convex subset of $X, x_{0} \in C$, and let $n_{0}$ be a positive integer. Suppose that $F: C \rightarrow C$ is $\psi$-convex-power condensing about $x_{0}$ and $n_{0}$. If $F$ is ws-compact and $F(C)$ is bounded, then $F$ has a fixed point in $C$.

Proof. Let

$$
\mathcal{F}=\left\{A \subseteq C, \overline{\mathrm{CO}}(A)=A, x_{0} \in A \text { and } F(A) \subseteq A\right\}
$$

The set $\mathcal{F}$ is nonempty since $C \in \mathcal{F}$. Set $M=\bigcap_{A \in \mathcal{F}} A$. Now, we show that for any positive integer $n$ we have

$$
M=\overline{\mathrm{co}}\left(F^{\left(n, x_{0}\right)}(M) \cup\left\{x_{0}\right\}\right)
$$

$(p(n))$ 
To see this, we proceed by induction. Clearly $M$ is a closed convex subset of $C$ and $F(M) \subseteq$ $M$. Thus, $M \in \mathcal{F}$. This implies $\overline{\mathrm{co}}\left(F(M) \cup\left\{x_{0}\right\}\right) \subseteq M$. Hence, $F\left(\overline{\mathrm{co}}\left(F(M) \cup\left\{x_{0}\right\}\right)\right) \subseteq F(M) \subseteq$ $\overline{\mathrm{co}}\left(F(M) \cup\left\{x_{0}\right\}\right)$. Consequently, $\overline{\mathrm{co}}\left(F(M) \cup\left\{x_{0}\right\}\right) \in \mathcal{F}$. Hence, $M \subseteq \overline{\mathrm{CO}}\left(F(M) \cup\left\{x_{0}\right\}\right)$. As a result $\overline{\mathrm{CO}}\left(F(M) \cup\left\{x_{0}\right\}\right)=M$. This shows that $(D(1))$ holds. Let $n$ be fixed, and suppose that $(\mathcal{D}(n))$ holds. This implies $F^{\left(n+1, x_{0}\right)}(M)=F\left(\overline{\mathrm{co}}\left(F^{\left(n, x_{0}\right)}(M) \cup\left\{x_{0}\right\}\right)=F(M)\right.$. Consequently,

$$
\overline{\mathrm{CO}}\left(F^{\left(n+1, x_{0}\right)}(M) \cup\left\{x_{0}\right\}\right)=\overline{\mathrm{CO}}\left(F(M) \cup\left\{x_{0}\right\}\right)=M .
$$

As a result

$$
\overline{\mathrm{CO}}\left(F^{\left(n_{0}, x_{0}\right)}(M) \cup\left\{x_{0}\right\}\right)=M
$$

Notice $F(C)$ is bounded implies that $M$ is bounded. Using the properties of the measure of weak noncompactness, we get

$$
\psi(M)=\psi\left(\overline{\mathrm{CO}}\left(F^{\left(n_{0}, x_{0}\right)}(M) \cup\left\{x_{0}\right\}\right)\right)=\psi\left(F^{\left(n_{0}, x_{0}\right)}(M)\right)
$$

which yields that $M$ is weakly compact. Now, we show that $F(M)$ is relatively compact. To see this, consider a sequence $\left(y_{n}\right)_{n \in \mathbb{N}}$ in $F(M)$. For each $n \in \mathbb{N}$, there exists $x_{n} \in M$ with $y_{n}=F x_{n}$. Now, the Eberlein- $\widetilde{S}$ mulian theorem [9, page 549] guarantees that there exists a subsequence $S$ of $\mathbb{N}$ so that $\left(x_{n}\right)_{n \in S}$ is a weakly convergent sequence. Since $F$ is ws-compact, then $\left(F x_{n}\right)_{n \in S}$ has a strongly convergent subsequence. Thus, $F(M)$ is relatively compact. Keeping in mind that $F(M) \subseteq M$, the result follows from Schauder's fixed point theorem.

As an easy consequence of Theorem 2.1, we recapture [10, Theorem 3.1].

Corollary 2.2. Let $X$ be a Banach space, and let $\psi$ be a regular and set additive measure of weak noncompactness on $X$. Let $C$ be a nonempty closed convex subset of $X$. Assume that $F: C \rightarrow C$ is ws-compact and $F(C)$ is bounded. If $F$ is $\psi$-condensing, that is, $\psi(F(M))<\psi(M)$, whenever $M$ is a bounded nonweakly compact subset of $C$, then $F$ has a fixed point.

Theorem 2.3. Let X be a Banach space, and let $\psi$ a measure of weak noncompactness on X. Let $C$ be a closed, convex subset of $X, U$ an open subset of $C$, and $p \in U$. Assume that $F: X \rightarrow X$ is ws-compact and $\psi$-convex-power condensing about $p$ and $n_{0}$. If $F(\bar{U}) \subseteq C$ and $F(\bar{U})$ is bounded, then either

(i) F has a fixed point in $\bar{U}$, or

(ii) there is a $u \in \partial U$ (the boundary of $U$ in $C$ ) and $\lambda \in(0,1)$ with $u=\lambda F(u)+(1-\lambda) p$.

Proof. Suppose that (ii) does not hold and $F$ has no fixed points on $\partial U$ (otherwise, we are finished). Then, $u \neq \lambda F(u)+(1-\lambda) p$ for $u \in \partial U$ and $\lambda \in[0,1]$. Consider

$$
A:=\{x \in \bar{U}: x=t F(x)+(1-t) p \text { for some } t \in[0,1]\} .
$$

Now, $A \neq \emptyset$ since $p \in U$. In addition, the continuity of $F$ implies that $A$ is closed. Notice that

$$
A \cap \partial U=\emptyset,
$$


therefore, by Urysohn's lemma, there exists a continuous $\mu: \bar{U} \rightarrow[0,1]$ with $\mu(A)=1$ and $\mu(\partial U)=0$. Let

$$
N(x)= \begin{cases}\mu(x) F(x)+(1-\mu(x)) p, & x \in \bar{U}, \\ p, & C \backslash \bar{U} .\end{cases}
$$

It is immediate that $N: C \rightarrow C$ is continuous. Now we show that $N$ is ws-compact. To see this, let $\left(x_{n}\right)_{n \in \mathbb{N}}$ be a sequence in $C$ which converges weakly to some $x \in C$. Without loss of generality, we may take $\left(x_{n}\right)_{n \in \mathbb{N}}$ in $\bar{U}$. Notice that $\left(\mu\left(x_{n}\right)\right)_{n \in \mathbb{N}}$ is a sequence in $[0,1]$. Hence, by extracting a subsequence if necessary, we may assume that $\left(\mu\left(x_{n}\right)\right)_{n \in \mathbb{N}}$ converges to some $\lambda \in[0,1]$. On the other hand, since $F$ is ws-compact, then there exists a subsequence $S$ of $\mathbb{N}$ so that $\left(F x_{n}\right)_{n \in S}$ converges strongly to some $y \in C$. Consequently, the sequence $\left(N x_{n}\right)_{n \in S}$ converges strongly to $\lambda y+(1-\lambda) p$. This proves that $N$ is ws-compact. Our next task is to show that $N$ is $\psi$-convex-power condensing about $p$ and $n_{0}$. To see this, let $S$ be a bounded subset of $C$. Clearly

$$
N(S) \subseteq \overline{\mathrm{co}}(F(S) \cup\{p\})
$$

By induction, note for all positive integer $n$, we have

$$
N^{(n, p)}(S) \subseteq \overline{c o}\left(F^{(n, p)}(S) \cup\{p\}\right)
$$

Indeed, fix an integer $n \geq 1$ and suppose that (2.9) holds. Then,

$$
\begin{aligned}
N^{(n+1, p)}(S) & =N\left(\overline{\mathrm{co}}\left(N^{(n, p)}(S) \cup\{p\}\right)\right) \\
& \subseteq N\left(\overline{\mathrm{CO}}\left(F^{(n, p)}(S) \cup\{p\}\right)\right) \\
& \subseteq \overline{\mathrm{co}}\left(F\left(\overline{\mathrm{co}}\left(F^{(n, p)}(S) \cup\{p\}\right)\right) \cup\{p\}\right) \\
& =\overline{\mathrm{co}}\left(F^{(n+1, p)}(S) \cup\{p\}\right) .
\end{aligned}
$$

In particular, we have

$$
N^{\left(n_{0}, p\right)}(S) \subseteq \overline{\mathrm{co}}\left(F^{\left(n_{0}, p\right)}(S) \cup\{p\}\right) .
$$

Thus,

$$
\psi\left(N^{\left(n_{0}, p\right)}(S)\right) \leq \psi\left(\overline{\mathrm{CO}}\left(F^{\left(n_{0}, p\right)}(S) \cup\{p\}\right)\right)=\psi\left(\left(F^{\left(n_{0}, p\right)}(S)\right)<\psi(S) .\right.
$$

This proves that $N$ is $\psi$-convex-power condensing about $p$ and $n_{0}$. Theorem 2.1 guarantees the existence of $x \in C$ with $x=N(x)$. Notice that $x \in U$ since $p \in U$. Thus, $x=\mu(x) F(x)+$ $(1-\mu(x)) p$. As a result, $x \in A$, and therefore $\mu(x)=1$. This implies that $x=F(x)$. 
Remark 2.4. Theorem 2.3 is a sharpening of [10, Theorem 4.1].

Lemma 2.5 (see [11]). Let $Q$ be a closed convex subset of a Banach space $X$ with $0 \in \operatorname{int}(Q)$. Let $\mu$ be the Minkowski functional defined by

$$
\mu(x)=\inf \{\lambda>0: x \in \lambda Q\},
$$

for all $x \in X$. Then,

(i) $\mu$ is nonnegative and continuous on $X$.

(ii) For all $\lambda \geq 0$ we have $\mu(\lambda x)=\lambda \mu(x)$.

(iii) $\mu(x)=1$ if and only if $x \in \partial Q$.

(iv) $0 \leq \mu(x)<1$ if and only if $x \in \operatorname{int}(Q)$.

(v) $\mu(x)>1$ if and only if $x \notin Q$.

Lemma 2.6. Let $X$ be a Banach space, $\psi$ a set additive measure of weak noncompactness on $X$, and $Q$ a closed convex subset of $X$ with $0 \in \operatorname{int}(Q)$. Let $\mu$ be the Minkowski functional defined in Lemma 2.5, and, $r$ be the map defined on $X$ by

$$
r(x)=\frac{x}{\max \{1, \mu(x)\}} \text { for } x \in X .
$$

Then,

(i) $r$ is continuous, $r(X) \subseteq Q$ and $r(x)=x$ for all $x \in Q$.

(ii) For any subset $A$ of $\mathrm{X}$ we have $r(A) \subseteq \operatorname{co}(A \cup\{0\})$.

(iii) For any bounded subset $A$ of $X$ we have $\psi(r(A)) \leq \psi(A)$.

Proof. (i) The continuity of $r$ follows immediately from Lemma 2.5(i). Now, let $x \in X$. Using Lemma 2.5(ii), we get

$$
\mu(r(x))=\frac{\mu(x)}{\max \{1, \mu(x)\}} \leq 1
$$

This implies that $r(x) \in Q$. The last statement follows easily from Lemma 2.5(v). Now, we prove (ii). To this end, let $A$ be a subset of $X$, and let $x \in A$. Then,

$$
\begin{aligned}
r(x)=\frac{x}{\max \{1, \mu(x)\}} & =\frac{1}{\max \{1, \mu(x)\}} x+\left(1-\frac{1}{\max \{1, \mu(x)\}}\right) 0 \\
& \in \operatorname{co}(A \cup\{0\}) .
\end{aligned}
$$

Thus, $r(A) \subseteq \operatorname{co}(A \cup\{0\})$. Using the properties of a measure of weak noncompactness, we get

$$
\psi(r(A)) \leq \psi(\operatorname{co}(A \cup\{0\}))=\psi(A \cup\{0\})=\psi(A) .
$$

This proves (iii). 
Theorem 2.7. Let $X$ be a Banach space, and let $\psi$ a regular set additive measure of weak noncompactness on $X$. Let $Q$ be a closed convex subset of $X$ with $0 \in Q$, and let $n_{0}$ a positive integer. Assume that $F: X \rightarrow X$ is ws-compact and $\psi$-convex-power condensing about 0 and $n_{0}$ and $F(Q)$ is bounded and

$$
\begin{aligned}
& \text { if }\left\{\left(x_{j}, \lambda_{j}\right)\right\} \text { is a sequence in } \partial Q \times[0,1] \text { converging to }(x, \lambda) \text { with } \\
& x=\lambda F(x) \text { and } 0<\lambda<1 \text {, then } \lambda_{j} F\left(x_{j}\right) \in Q \text { for } j \text { sufficiently large }
\end{aligned}
$$

holding. Also, suppose the following condition holds:

there exists a continuous retraction $r: X \longrightarrow Q$ with $r(z) \in \partial Q$ for $z \in X \backslash Q$

and $r(D) \subseteq \operatorname{co}(D \cup\{0\})$ for any bounded subset $D$ of $X$.

Then, F has a fixed point.

Proof. Let $r: X \rightarrow Q$ be as described in (2.19). Consider

$$
B=\{x \in X: x=\operatorname{Fr}(x)\}
$$

We first show that $B \neq \emptyset$. To see this, consider $r F: Q \rightarrow Q$. First, notice that $r F(Q)$ is bounded since $F(Q)$ is bounded and $r(F(Q)) \subseteq \operatorname{co}(F(Q) \cup\{0\})$. Clearly, $r F$ is continuous, since $F$ and $r$ are continuous. Now, we show that $r F$ is ws-compact. To see this, let $\left(x_{n}\right)_{n \in \mathbb{N}}$ be a sequence in $Q$ which converges weakly to some $x \in Q$. Since $F$ is ws-compact, then there exists a subsequence $S$ of $\mathbb{N}$ so that $\left(F x_{n}\right)_{n \in S}$ converges strongly to some $y \in X$. The continuity of $r$ guarantees that the sequence $\left(r F x_{n}\right)_{n \in S}$ converges strongly to $r y$. This proves that $r F$ is ws-compact. Our next task is to show that $r F$ is $\psi$-convex-power condensing about 0 and $n_{0}$. To do so, let $A$ be a subset of $Q$. In view of (2.19), we have

$$
(r F)^{(1,0)}(A)=r F(A)=r F^{(1,0)}(A) \subseteq \overline{\mathrm{CO}}\left(F^{(1,0)}(A) \cup\{0\}\right) .
$$

Hence,

$$
\begin{aligned}
(r F)^{(2,0)}(A) & =r F\left(\overline{\mathrm{CO}}\left((r F)^{(1,0)}(A) \cup\{0\}\right)\right) \\
& =r F\left(\overline{\mathrm{CO}}\left(r F^{(1,0)}(A) \cup\{0\}\right)\right) \\
& \subseteq r F\left(\overline{\mathrm{CO}}\left(F^{(1,0)}(A) \cup\{0\}\right)\right) \\
& =r F^{(2,0)}(A),
\end{aligned}
$$


and by induction

$$
\begin{aligned}
(r F)^{\left(n_{0}, 0\right)}(A) & =r F\left(\overline{\mathrm{CO}}\left((r F)^{\left(n_{0}-1,0\right)}(A) \cup\{0\}\right)\right) \\
& \subseteq r F\left(\overline{\mathrm{CO}}\left(r F^{\left(n_{0}-1,0\right)}(A) \cup\{0\}\right)\right) \\
& \subseteq r F\left(\overline{\mathrm{CO}}\left(F^{\left(n_{0}-1,0\right)}(A) \cup\{0\}\right)\right) \\
& =r F^{\left(n_{0}, 0\right)}(A) .
\end{aligned}
$$

Taking into account the fact that $F$ is $\psi$-convex-power condensing about 0 and $n_{0}$ and using (2.19), we get

$$
\begin{aligned}
\psi\left((r F)^{\left(n_{0}, 0\right)}(A)\right) & \leq \psi\left(r F^{\left(n_{0}, 0\right)}(A)\right) \leq \psi\left(\operatorname{co}\left(F^{\left(n_{0}, 0\right)}(A) \cup\{0\}\right)\right) \\
& \leq \psi\left(F^{\left(n_{0}, 0\right)}(A)\right)<\psi(A),
\end{aligned}
$$

whenever $\psi(A)>0$. Invoking Theorem 2.1, we infer that there exists $y \in Q$ with $r F(y)=y$. Let $z=F(y)$, so $\operatorname{Fr}(z)=\operatorname{Fr}(F(y))=F(y)=z$. Thus, $z \in B$ and $B \neq \emptyset$. In addition, $B$ is closed, since $F r$ is continuous. Moreover, we claim that $B$ is compact. To see this, first notice

$$
B \subseteq F r(B) \subseteq F\left(B^{\prime}\right)=F^{(1,0)}\left(B^{\prime}\right)
$$

where $B^{\prime}=\overline{\mathrm{CO}}(B \cup\{0\})$. Thus,

$$
\begin{aligned}
B & \subseteq F r(B) \subseteq F r\left(F\left(B^{\prime}\right)\right) \\
& \subseteq F\left(\overline{\mathrm{CO}}\left(F\left(B^{\prime}\right) \cup\{0\}\right)\right) \\
& =F^{(2,0)}\left(B^{\prime}\right),
\end{aligned}
$$

and by induction

$$
\begin{aligned}
B & \subseteq F r(B) \subseteq F r\left(F^{\left(n_{0}-1,0\right)}\left(B^{\prime}\right)\right) \\
& \subseteq F\left(\overline{\mathrm{Co}}\left(F^{\left(n_{0}-1,0\right)}\left(B^{\prime}\right) \cup\{0\}\right)\right) \\
& =F^{\left(n_{0}, 0\right)}\left(B^{\prime}\right),
\end{aligned}
$$

Now, if $\psi(B) \neq 0$, then

$$
\psi(B) \leq \psi\left(F^{\left(n_{0}, 0\right)}\left(B^{\prime}\right)\right)<\psi\left(B^{\prime}\right)=\psi(B)
$$

which is a contradiction. Thus, $\psi(B)=0$ and so $B$ is relatively weakly compact. Now, (2.19) guarantees that $r(B)$ is relatively weakly compact. Now, we show that $\operatorname{Fr}(B)$ is relatively 
compact. To see this, let $\left(y_{n}\right)_{n \in \mathbb{N}}$ be a sequence in $\operatorname{Fr}(B)$. For each $n \in \mathbb{N}$, there exists $x_{n} \in r(B)$ with $y_{n}=F x_{n}$. Since $r(B)$ is relatively weakly compact, then, by extracting a subsequence if necessary, we may assume that $\left(x_{n}\right)_{n \in \mathbb{N}}$ is a weakly convergent sequence. Now, $F$ is ws-compact implies that $\left(y_{n}\right)_{n \in \mathbb{N}}$ has a strongly convergent subsequence. This proves that $\operatorname{Fr}(B)$ is relatively compact. From (2.25), it readily follows that $B$ is relatively compact. Consequently, $B=\bar{B}$ is compact. We now show that $B \cap Q \neq \emptyset$. To do this, we argue by contradiction. Suppose that $B \cap Q=\emptyset$. Then, since $B$ is compact and $Q$ is closed, there exists $\delta>0$ with $\operatorname{dist}(B, Q)>\delta$. Choose $N \in\{1,2, \ldots\}$ such that $N \delta>1$. Define

$$
U_{i}=\left\{x \in X: d(x, Q)<\frac{1}{i}\right\} \text { for } i \in\{N, N+1, \ldots\}
$$

here $d(x, Q)=\inf \{\|x-y\|: y \in Q\}$. Fix $i \in\{N, N+1, \ldots\}$. Since $\operatorname{dist}(B, Q)>\delta$, then $B \cap \overline{U_{i}}=\emptyset$. Now, we show that $F r: \overline{U_{i}} \rightarrow X$ is ws-compact. To see this, let $\left(x_{n}\right)_{n \in \mathbb{N}}$ be a weakly convergent sequence in $\overline{U_{i}}$. Then, the set $S:=\left\{x_{n}: n \in \mathbb{N}\right\}$ is relatively weakly compact and so $\psi(S)=0$. In view of (2.19), we infer that $\psi(r(S))=0$ and so $r(S)$ is relatively weakly compact. By extracting a subsequence if necessary, we may assume that $\left(r x_{n}\right)_{n \in \mathbb{N}}$ is weakly convergent. Now, $F$ is ws-compact implies that $\left(F r x_{n}\right)_{n \in \mathbb{N}}$ has a strongly convergent subsequence. This proves that $F r$ is ws-compact. Our next task is to show that $F r$ is $\psi$-convex-power condensing about 0 and $n_{0}$. To see this, let $A$ be a bounded subset of $\overline{U_{i}}$ and set $A^{\prime}=\overline{\mathrm{co}}(A \cup\{0\})$. Then, keeping in mind (2.19), we obtain

$$
\begin{aligned}
&(F r)^{(1,0)}(A) \subseteq F\left(A^{\prime}\right), \\
&(F r)^{(2,0)}(A)=F r\left(\overline{\mathrm{co}}\left((F r)^{(1,0)}(A) \cup\{0\}\right)\right) \\
& \subseteq F r\left(\overline{\mathrm{co}}\left(F\left(A^{\prime}\right) \cup\{0\}\right)\right) \\
& \subseteq F\left(\overline{\mathrm{co}}\left(F\left(A^{\prime}\right) \cup\{0\}\right)\right) \\
&=F^{(2,0)}\left(A^{\prime}\right),
\end{aligned}
$$

and by induction,

$$
\begin{aligned}
(F r)^{\left(n_{0}, 0\right)}(A) & =\operatorname{Fr}\left(\overline{\mathrm{CO}}\left((F r)^{\left(n_{0}-1,0\right)}(A) \cup\{0\}\right)\right) \\
& \subseteq \operatorname{Fr}\left(\overline{\mathrm{CO}}\left(F^{\left(n_{0}-1,0\right)}\left(A^{\prime}\right) \cup\{0\}\right)\right) \\
& \subseteq F\left(\overline{\mathrm{CO}}\left(F^{\left(n_{0}-1,0\right)}\left(A^{\prime}\right) \cup\{0\}\right)\right) \\
& =F^{\left(n_{0}, 0\right)}\left(A^{\prime}\right) .
\end{aligned}
$$

Thus,

$$
\psi\left((F r)^{\left(n_{0}, 0\right)}(A)\right) \leq \psi\left(F^{\left(n_{0}, 0\right)}\left(A^{\prime}\right)\right)<\psi\left(A^{\prime}\right)=\psi(A)
$$


whenever $\psi(A) \neq 0$. Applying Theorem 2.3 to $F r: \overline{U_{i}} \rightarrow X$, we may deduce that there exists $\left(y_{i}, \lambda_{i}\right) \in \partial U_{i} \times(0,1)$ with $y_{i}=\lambda_{i} F r\left(y_{i}\right)$. Notice in particular since $y_{i} \in \partial U_{i} \times(0,1)$ that

$$
\lambda_{i} \operatorname{Fr}\left(y_{i}\right) \notin Q \quad \text { for } i \in\{N, N+1, \ldots\}
$$

We now consider

$$
D=\{x \in X: x=\lambda F r(x) \text {, for some } \lambda \in[0,1]\} .
$$

Clearly, $D$ is closed since $F$ and $r$ are continuous. Now, we claim that $D$ is compact. To see this, first notice

$$
D \subseteq \operatorname{Fr}(D) \cup\{0\}
$$

Thus,

$$
D \subseteq F r(D) \cup\{0\} \subseteq \operatorname{Fr}(\overline{\mathrm{CO}}(F r(D) \cup\{0\})) \cup\{0\}=(F r)^{(2,0)} \cup\{0\},
$$

and by induction

$$
D \subseteq F r(D) \cup\{0\} \subseteq F r\left(\overline{\mathrm{CO}}\left((F r)^{\left(n_{0}-1,0\right)}(D) \cup\{0\}\right)\right) \cup\{0\}=(F r)^{\left(n_{0}, 0\right)} \cup\{0\},
$$

consequently

$$
\psi(D) \leq \psi\left((F r)^{\left(n_{0}, 0\right)} \cup\{0\}\right) \leq \psi\left((F r)^{\left(n_{0}, 0\right)}\right)
$$

Since $F r$ is $\psi$-convex-power condensing about 0 and $n_{0}$, then $\psi(D)=0$, and so $D$ is relatively weakly compact. Now, (2.19) guarantees that $r(D)$ is relatively weakly compact. Now, we show that $\operatorname{Fr}(D)$ is relatively compact. To see this, let $\left(y_{n}\right)_{n \in \mathbb{N}}$ be a sequence in $F(D)$. For each $n \in \mathbb{N}$, there exists $x_{n} \in r(D)$ with $y_{n}=F x_{n}$. Since $r(D)$ is relatively weakly compact then, by extracting a subsequence if necessary, we may assume that $\left(x_{n}\right)_{n \in \mathbb{N}}$ is a weakly convergent sequence. Now, $F$ is ws-compact implies that $\left(y_{n}\right)_{n \in \mathbb{N}}$ has a strongly convergent subsequence. This proves that $\operatorname{Fr}(D)$ is relatively compact. From (2.35), it readily follows that $D$ is relatively compact. Consequently, $D=\bar{D}$ is compact. Then, up to a subsequence, we may assume that $\lambda_{i} \rightarrow \lambda^{*} \in[0,1]$ and $y_{i} \rightarrow y^{*} \in \partial U_{i}$. Hence, $\lambda_{i} F r\left(y_{i}\right) \rightarrow \lambda^{*} F r\left(y^{*}\right)$, and therefore $y^{*}=$ $\lambda^{*} \operatorname{Fr}\left(y^{*}\right)$. Notice $\lambda^{*} \operatorname{Fr}\left(y^{*}\right) \notin Q$ since $y^{*} \in \partial U_{i}$. Thus, $\lambda^{*} \neq 1$ since $B \cap Q=\emptyset$. From assumption (2.18), it follows that $\lambda_{i} \operatorname{Fr}\left(y_{i}\right) \in Q$ for $j$ sufficiently large, which is a contradiction. Thus, $B \cap Q \neq \emptyset$, so there exists $x \in Q$ with $x=F r(x)$, that is, $x=F x$.

Remark 2.8. If $0 \in \operatorname{int}(Q)$ then we can choose $r: X \rightarrow Q$ in the statement of Theorem 2.7 as in Lemma 2.6. Clearly $r(z) \in \partial Q$ for $z \in X \backslash Q$ and $r(D) \subseteq \operatorname{co}(D \cup\{0\})$ for any bounded subset $D$ of $X$. 
Corollary 2.9. Let $X$ be a Banach space, $\psi$ a regular set additive measure of weak noncompactness on $X$, and $Q$ a closed convex subset of $X$ with $0 \in Q$. Assume that $F: X \rightarrow X$ is ws-compact and $\psi$-convex-power condensing about 0 and $n_{0}$, and assume that (2.19) holds. If $F(Q)$ is bounded and $F(\partial Q) \subseteq Q$ (the condition of Rothe type), then $F$ has a fixed point in $Q$.

In the light of Remark 2.8, we have the following result.

Corollary 2.10. Let $X$ be a Banach space, $\psi$ a regular set additive measure of weak noncompactness on $X$ and $Q$ a closed convex subset of $X$ with $0 \in \operatorname{int}(Q)$. Assume $F: X \rightarrow X$ is ws-compact and $\psi$-convex-power condensing about 0 and $n_{0}$. If $F(Q)$ is bounded and $F(\partial Q) \subseteq Q$, then $F$ has a fixed point in $Q$.

Theorem 2.11. Let $Q$ be a closed convex set in a Banach space $X, 0 \in \operatorname{int}(Q)$. Assume $F: X \rightarrow X$ is ws-compact and $\psi$-convex-power condensing about 0 and $n_{0}$. If $F(Q)$ is bounded and

$$
\|F x-x\|^{2} \geq\|F x\|^{2}-\|x\|^{2}, \quad \forall x \in \partial Q,
$$

(the condition of Altman type), then $F$ has a fixed point in $Q$.

Proof. Let $r: X \rightarrow Q$ be as described in Lemma 2.6. As in the proof of Theorem 2.7, there exists $z \in Q$ such that $z=r F(z)$. If $F z \in Q$, then $z=r F z=F z$, and we are done. If $F z \notin Q$, by Lemma 2.5, we have $\mu(F z)>1$. Thus, $z=r F z=(1 / \mu(F z)) F z$. Letting $\lambda=\mu(F z)$, then $F z=\lambda z$. Consequently,

$$
\|\mathrm{F} z-z\|^{2}=(\lambda-1)^{2}\|z\|^{2} \geq\|F z\|^{2}-\|z\|^{2}=\left(\lambda^{2}-1\right)\|z\|^{2} .
$$

As a result $(\lambda-1)^{2} \geq\left(\lambda^{2}-1\right)$. This contradicts the fact that $\lambda>1$. Therefore, $F$ has a fixed point.

Corollary 2.12. Let $Q$ be a closed convex set in a Banach space $X, 0 \in \operatorname{int}(Q)$. Assume that $F: X \rightarrow$ $X$ is ws-compact and $\psi$-convex-power condensing about 0 and $n_{0}$. If $F(Q)$ is bounded and one of the following conditions are satisfied:

(i) $\|F x\| \leq\|x\|$, for all $x \in \partial Q$ (the condition of Rothe type),

(ii) $\|x-F x\| \geq\|F x\|$, for all $x \in \partial Q$ (the condition of Petryshyn type).

Then, $F$ has a fixed point in $Q$.

Remark 2.13. In Theorem 2.7 we need $F: X \rightarrow X \psi$-convex-power condens-ing about 0 and $n_{0}$ : However, In Theorem 2.7 the condition $F: X \rightarrow X$ ws-compact can be replaced by $F$ : $Q \rightarrow X$ ws-compact. This comment also applies to Corollaries 2.9, 2.10, Theorem 2.11, and Corollary 2.12.

In the following example, we give a broad class of ws-compact mappings which are not sequentially weakly continuous. 
Example 2.14. Let $g:(0,1) \times \mathbb{R} \rightarrow \mathbb{R}$ be a function satisfying Carathéodory conditions, that is, $g$ is Lebesgue measurable in $x$ for each $y \in \mathbb{R}$ and continuous in $y$ for each $x \in(0,1)$. Additionally, we assume that

$$
|g(x, y)| \leq a(x)+b|y|
$$

for all $(x, y) \in(0,1) \times \mathbb{R}$, where $a(x)$ is a nonnegative function Lebesgue integrable on the interval $(0,1)$ and $b \geq 0$. Let us consider the so-called superposition operator $N_{g}$, generated by the function $g$, which to every function $u$ defined on the interval $(0,1)$ assigns the function $N_{g} u$ given by the formula

$$
\left(N_{g} u\right)(x)=g(x, u(x)), \quad x \in(0,1) .
$$

Let $L^{1}=L^{1}(0,1)$ denote the space of functions $u:(0,1) \rightarrow \mathbb{R}$ which are Lebesgue integrable, equipped with the standard norm. It was shown [12] that under the above-quoted assumptions the superposition operator $N_{g}$ maps continuously the space $L^{1}$ into itself. Define the functional

$$
\phi(u)=\int_{0}^{1} N_{g} u(x) d x=\int_{0}^{1} g(x, u(x)) d x
$$

for $u \in L^{1}$. Notice that $\phi=K N_{g}$, where $K$ is the linear functional defined on $L^{1}$ by

$$
K(u)=\int_{0}^{1} u(x) d x, \quad u \in L^{1} .
$$

Clearly, $K$ is continuous with norm $\|K\| \leq 1$. Thus, $\phi$ is continuous. Now, we show that $\phi$ is ws-compact. To see this, let $\left(u_{n}\right)$ be a weakly convergent sequence of $L^{1}$. Using (2.41), we have for any for any subset $D$ of $(0,1)$ that

$$
\int_{D}\left|N_{g} u_{n}(x)\right| d x \leq \int_{D} a(x) d t+b \int_{D}\left|u_{n}(x)\right| d x
$$

Taking into account the fact the sequence $\left(u_{n}\right)$ is weakly convergent and that any set consisting of one element is weakly compact and using Corollary 11 in [13, page 294], we get

$$
\begin{gathered}
\lim _{|D| \rightarrow 0} \int_{D} a(x) d x=0 \\
\lim _{|D| \rightarrow 0} \int_{D}\left|u_{n}(x)\right| d x=0
\end{gathered}
$$

uniformly in $n$, where $|D|$ is the Lebesgue measure of $D$. Combining (2.45) and(2.46), we arrive at

$$
\lim _{|D| \rightarrow 0} \int_{D}\left|N_{g} u_{n}(x)\right| d x=0
$$


uniformly in $n$. Applying Corollary 11 in [13, page 294] once again, we infer that $N_{g}\left(u_{n}\right)$ has a weakly convergent subsequence, say $N_{g}\left(u_{n_{k}}\right)$. Let $u$ be the weak limit of $\left(N_{g}\left(u_{n_{k}}\right)\right)$. Hence,

$$
\int_{0}^{1} N_{g}\left(u_{n_{k}}\right)(x) v(x) d x \rightarrow \int_{0}^{1} u(x) v(x) d x,
$$

for all $v \in L^{\infty}(0,1)$. In particular, we have

$$
\int_{0}^{1} g\left(x, u_{n_{k}}(x)\right) d x \longrightarrow \int_{0}^{1} u(x) d x
$$

Consequently, the sequence $\left(\phi u_{n_{k}}\right)$ is convergent. This proves that $\phi$ is ws-compact. However, $\phi$ is not weakly sequentially continuous unless $\phi$ is linear with respect to the second variable (see $[14,15])$.

\section{References}

[1] J. Jachymski, "On Isac's fixed point theorem for selfmaps of a Galerkin cone," Annales des Sciences Mathématiques du Québec, vol. 18, no. 2, pp. 169-171, 1994.

[2] J. García-Falset, "Existence of fixed points and measures of weak noncompactness," Nonlinear Analysis: Theory, Methods \& Applications, vol. 71, no. 7-8, pp. 2625-2633, 2009.

[3] K. Latrach and M. A. Taoudi, "Existence results for a generalized nonlinear Hammerstein equation on $L^{1}$ spaces," Nonlinear Analysis: Theory, Methods \& Applications, vol. 66, no. 10, pp. 2325-2333, 2007.

[4] K. Latrach, M. A. Taoudi, and A. Zeghal, "Some fixed point theorems of the Schauder and the Krasnosel'skii type and application to nonlinear transport equations," Journal of Differential Equations, vol. 221, no. 1, pp. 256-271, 2006.

[5] M. Aziz Taoudi, "Integrable solutions of a nonlinear functional integral equation on an unbounded interval," Nonlinear Analysis: Theory, Methods E Applications, vol. 71, no. 9, pp. 4131-4136, 2009.

[6] J. Banaś and J. Rivero, "On measures of weak noncompactness," Annali di Matematica Pura ed Applicata, vol. 151, pp. 213-224, 1988.

[7] F. S. De Blasi, "On a property of the unit sphere in a Banach space," Bulletin Mathématique de la Société des Sciences Mathématiques de Roumanie, vol. 21, no. 3-4, pp. 259-262, 1977.

[8] J. X. Sun and X. Y. Zhang, "A fixed point theorem for convex-power condensing operators and its applications to abstract semilinear evolution equations," Acta Mathematica Sinica, vol. 48, no. 3, pp. 439-446, 2005 (Chinese).

[9] R. E. Edwards, Functional Analysis. Theory and Applications, Holt, Rinehart and Winston, 1965.

[10] A. Ben Amar and J. Garcia-Falset, "Fixed point theorems for 1-set weakly contractive and pseudocontractive operators on an unbounded domain," to appear in Portugaliae Mathematica.

[11] A. E. Taylor and D. C. Lay, Introduction to Functional Analysis, John Wiley \& Sons, New York, NY, USA, 2nd edition, 1980.

[12] M. A. Krasnosel'skii, "On the continuity of the operator $F u(x)=f(x, u(x))$," Doklady Akademii Nauk SSSR, vol. 77, pp. 185-188, 1951 (Russian).

[13] N. Dunford and J. T. Schwartz, Linear Operators, Part I: General Theory, John Wiley \& Sons, New York, NY, USA, 1988.

[14] R. Černý, S. Hencl, and J. Kolár̆, "Integral functionals that are continuous with respect to the weak topology on $W_{0}^{1, p}(\Omega), "$ Nonlinear Analysis: Theory, Methods $\mathcal{E}$ Applications, vol. 71, no. 7-8, pp. 27532763, 2009.

[15] S. Hencl, J. Kolář, and O. Pangrác, "Integral functionals that are continuous with respect to the weak topology on $W_{0}^{1, p}(0,1)$, " Nonlinear Analysis: Theory, Methods E Applications, vol. 63, no. 1, pp. 81-87, 2005. 